\title{
Design of an Optimal Preview Controller for a Class of Linear Discrete-Time Descriptor Systems
}

\author{
Fucheng Liao, Zhihua Xue, and Jiang Wu \\ School of Mathematics and Physics, University of Science and Technology Beijing, Beijing 100083, China \\ Correspondence should be addressed to Fucheng Liao; fcliao@ustb.edu.cn
}

Received 23 January 2017; Accepted 8 May 2017; Published 12 June 2017

Academic Editor: Mohammad D. Aliyu

Copyright ( 2017 Fucheng Liao et al. This is an open access article distributed under the Creative Commons Attribution License, which permits unrestricted use, distribution, and reproduction in any medium, provided the original work is properly cited.

\begin{abstract}
The preview control problem of a class of linear discrete-time descriptor systems is studied. Firstly, the descriptor system is decomposed into a normal system and an algebraic equation by the method of the constrained equivalent transformation. Secondly, by applying the first-order forward difference operator to the state equation, combined with the error equation, the error system is obtained. The tracking problem is transformed into the optimal preview control problem of the error system. Finally, the optimal controller of the error system is obtained by using the related results and the optimal preview controller of the original system is gained. In this paper, we propose a numerical simulation method for descriptor systems. The method does not depend on the restricted equivalent transformation.
\end{abstract}

\section{Introduction}

Preview control theory takes full advantage of future known reference signals or disturbance signals information to improve the dynamic response, to inhibit the disturbance, and to increase the tracking performance of the systems. The traditional method is to construct an auxiliary system (called error system) by combining the error equation and the difference equation. As a result, the tracking problem can be transformed into a regulation problem. By using known results [1] of optimal regulation theory, the optimal controller of the error system is obtained. Furthermore, the optimal preview controller of the original system is also obtained [2]. After more than 50 years' development, many methods have been proposed for preview controller designing [3-7]. In [3], for the linear discrete-time system with previewable reference signal and disturbance signal, the augmented error system is constructed by using the difference operator and the preview controller is designed. And the result is applied to the tracking problem of the generator control system. In [4], the augmented error system is constructed to solve the problem of designing preview controller for the continuous time system. In [5], the unified algorithm of linear system and $H_{\infty}$ preview control problem is presented by using the Hamiltonian matrix method, which is suitable for both continuous and discrete-time systems. In recent years, more attention has been paid to the applications of preview control theory [8-10]. Based on the least mean square algorithm of $-\mathrm{X}$ filter, [8] proposed a method to realize the preview feed-forward control by using the future wind speed information, which can adjust the rotor speed and reduce the load of the wind turbine. Reference [9] proposed an output-feedback $H_{\infty}$ preview controller and improved the antijamming performance and robustness of UAV flight control system.

Descriptor systems, also known as singular systems, are a class of dynamic systems. It contains not only the normal differential equations, but also algebraic equations. The study of descriptor system theory begins in the 1970s. After more than 40 years' development, it has gradually formed a complete theoretical system and method [1116]. Reference [11] systematically introduced the theory and method of the analysis and synthesis for descriptor system. Reference [12] studied the nonfragile $H_{\infty}$ control problem for a class of uncertain T-S fuzzy descriptor systems. And a state feedback controller with parameter uncertainties was designed. Reference [13] considered the adaptive observer 
design problem for a class of multi-input-multi-output linear descriptor systems. Reference [14] proposed an adaptive fault diagnosis observer to estimate the actuator fault for nonlinear descriptor systems. Reference [15] studied the observer problem of full and reduced dimensions for nonsquared descriptor systems with unknown inputs.

The research of preview control theory for descriptor systems begins in 2012. In [17], preview control theory was extended to descriptor systems. In [18], preview control theory for discrete-time descriptor systems with time-delay was studied. In [19], the theory was extended to continuous time conditions.

In this paper, the optimal preview control problem for discrete-time descriptor systems, with both reference signal and disturbance signal known, is studied. First, the system is decoupled into a normal equation and an algebraic equation. Then, by applying the first-order forward difference operator to the normal equation, a difference equation is obtained. Thus, the error system is constructed by combing the difference equation and the error equation. Finally, the optimal regulator for the error system is obtained, and as a result, the optimal preview controller for the original descriptor system is also gained. However, because there is a singular matrix in the original system, the closed-loop system cannot be directly simulated. The previous simulation work was figured out by the system after the limited equivalent transformation, rather than by the original system. Therefore, the main contribution in this paper is to design a more general simulation method for descriptor systems.

\section{Expression and Assumptions of the Problem}

Consider the discrete-time descriptor system:

$$
\begin{aligned}
E x(k+1) & =A x(k)+B u(k)+G d(k), \\
y(k) & =C x(k)+D u(k),
\end{aligned}
$$

where $x(k) \in R^{n}, u(k) \in R^{r}, y(k) \in R^{m}$, and $d(k) \in$ $R^{s}$ are the state vector, the input vector, the output vector, and the disturbance vector, respectively. $A, B, C, D, G$ are known constant matrices with appropriate dimensions. $E$ is a singular matrix with $0<\operatorname{rank}(E)=q<n$.

In this paper, only causal systems are discussed. First, it is assumed that system (1) is a causal system. Other necessary assumptions are as follows.

Assumption 1. Assume that $(E, A, B)$ is stabilizable and the matrix $\left[\begin{array}{cc}E-A & B \\ -C & D\end{array}\right]$ is of full row rank.

Assumption 2. Assume that $(E, A, C)$ is detectable.

Assumption 3. Assume that the preview length of the reference signal $r(k) \in R^{m}$ is $M_{r}$. That is, at each time $k$, the future values $r(k+1), r(k+2), \ldots, r\left(k+M_{r}\right)$, as well as the present and past values of the reference signal, are available. The future values of the reference signal are assumed to be unchanged after $r\left(k+M_{r}\right)$; namely,

$$
r(k+j)=r\left(k+M_{r}\right), \quad j=M_{r}+1, M_{r}+2, \ldots
$$

Assumption 4. Assume that the preview length of the disturbance signal $d(k) \in R^{s}$ is $M_{d}$. That is, at each time $k$, the future values $d(k+1), d(k+2), \ldots, d\left(k+M_{d}\right)$, as well as the present and past values of the disturbance signal, are available. The future values of the disturbance signal are assumed to be unchanged after $d\left(k+M_{d}\right)$; namely,

$$
d(k+j)=d\left(k+M_{d}\right), \quad j=M_{d}+1, M_{d}+2, \ldots
$$

The error vector is defined as follows:

$$
e(k)=y(k)-r(k) \text {. }
$$

Our target is to design an optimal preview controller with preview feed-forward compensation for system (1), so that the output $y(k)$ of the system can track the reference signal $r(k)$; that is,

$$
\lim _{k \rightarrow \infty} e(k)=\lim _{k \rightarrow \infty}(y(k)-r(k))=0 .
$$

Therefore, the performance index can be designed as

$$
J=\sum_{k=1}^{\infty}\left[e^{T}(k) Q_{e} e(k)+\Delta u^{T}(k) H \Delta u(k)\right],
$$

where the weight matrices satisfy $Q_{e}>0$ and $H>0$.

Remark 5. There are two benefits when introducing $\Delta u(k)$ to the performance index: (1), it is convenient to design the controller for the error system; (2) as a result, an integrator can be contained in the closed-loop system, and the static error can be eliminated by the integrator [2]. If the performance index function $J$ in (6) can be minimized by $\Delta u(k), u(k)$, as the input of system (1), can make the closedloop system satisfy the requirements.

\section{Limited Equivalent Transformation}

In order to make full use of the conclusions of optimal preview theory in normal system, system (1) needs to be changed into a normal system and an algebraic equation by limited equivalent transformation [11]. Since $0<\operatorname{rank}(E)=$ $q<n, E$ can be transformed into a diagonal form by primary transformation. That is, there exists nonsingular matrices $P_{1}$ and $Q_{1}$ such that $Q_{1} E P_{1}=\left[\begin{array}{cc}I_{q} & 0 \\ 0 & 0\end{array}\right] \stackrel{\text { def }}{=} \bar{E}$. For system (1), introducing a nonsingular linear transformation

$$
x(k)=P_{1} \bar{x}(k)
$$

and multiplying a nonsingular matrix $Q_{1}$ on both sides, we can get

$$
\begin{aligned}
\bar{E} \bar{x}(k+1) & =\bar{A} \bar{x}(k)+\bar{B} u(k)+\bar{G} d(k), \\
y(k) & =\bar{C} \bar{x}(k)+D u(k),
\end{aligned}
$$


where $\bar{x}(k)=\left[\begin{array}{l}\bar{x}_{1}(k) \\ \bar{x}_{2}(k)\end{array}\right], \bar{x}_{1}(k) \in R^{q}$ and $\bar{x}_{2}(k) \in R^{n-q}$. Then, (8) can be written as

$$
\begin{aligned}
\bar{x}_{1}(k+1)= & \bar{A}_{11} \bar{x}_{1}(k)+\bar{A}_{12} \bar{x}_{2}(k)+\bar{B}_{1} u(k) \\
& +\bar{G}_{1} d(k), \\
0= & \bar{A}_{21} \bar{x}_{1}(k)+\bar{A}_{22} \bar{x}_{2}(k)+\bar{B}_{2} u(k) \\
& +\bar{G}_{2} d(k), \\
y(k)= & \bar{C}_{1} \bar{x}_{1}(k)+\bar{C}_{2} x_{2}(k)+D u(k),
\end{aligned}
$$

where

$$
\begin{aligned}
Q_{1} A P_{1} & =\bar{A}=\left[\begin{array}{ll}
\bar{A}_{11} & \bar{A}_{12} \\
\bar{A}_{21} & \bar{A}_{22}
\end{array}\right], \\
Q_{1} B & =\bar{B}=\left[\begin{array}{l}
\bar{B}_{1} \\
\bar{B}_{2}
\end{array}\right], \\
Q_{1} G & =\bar{G}=\left[\begin{array}{l}
\bar{G}_{1} \\
\bar{G}_{2}
\end{array}\right], \\
C P_{1} & =\bar{C}=\left[\begin{array}{ll}
\bar{C}_{1} & \bar{C}_{2}
\end{array}\right] .
\end{aligned}
$$

Remark 6. The transformation above is called the limited equivalent transformation [11]. According to the existing conclusions in [11], the dynamic characteristics of the descriptor system, including the regularity, causality, stabilizability, and detectability, remain unchanged after the transformation. Therefore, these characteristics of system (1) can be obtained by studying system (8) or (9).

The so-called "system (1) is causal" means that the matrix $\bar{A}_{22}$ in system (9) is nonsingular. A necessary and sufficient condition for system (1) being regular is that there exists $s$, such that

$$
\operatorname{det}(s E-A) \neq 0
$$

holds.

Remark 7. Since

$$
\begin{aligned}
& \operatorname{det}(s E-A)=\operatorname{det}\left(s Q_{1}^{-1} \bar{E} P_{1}^{-1}-Q_{1}^{-1} \bar{A} P_{1}^{-1}\right) \\
& =\operatorname{det}\left(Q_{1}^{-1}\right) \operatorname{det}(s \bar{E}-\bar{A}) \operatorname{det}\left(P_{1}^{-1}\right) \\
& =\operatorname{det}\left(Q_{1}^{-1}\right) \operatorname{det}\left(s I-\bar{A}_{11}+\bar{A}_{12} \bar{A}_{22}^{-1} \bar{A}_{21}\right) \operatorname{det}\left(-\bar{A}_{22}\right) \operatorname{det}\left(P_{1}^{-1}\right),
\end{aligned}
$$

we can conclude that, for any $s$ being not equal to the characteristic value of $\bar{A}_{11}-\bar{A}_{12} \bar{A}_{22}^{-1} \bar{A}_{21}$, if $\bar{A}_{22}$ is nonsingular, $\operatorname{det}(s E-A) \neq 0$. Obviously, there are many $s$ satisfying the above condition. The above proof shows that the causality of system (1) ensures the regularity. Therefore, no other specific requirement for regularity in system (1) is needed.

Due to system (1) being causal, $\bar{A}_{22}$ in (9) is nonsingular. Then, according to the second equation in (9), we obtain

$$
\bar{x}_{2}(k)=-\bar{A}_{22}^{-1} \bar{A}_{21} \bar{x}_{1}(k)-\bar{A}_{22}^{-1} \bar{B}_{2} u(k)-\bar{A}_{22}^{-1} \bar{G}_{2} d(k) .
$$

Substituting (13) into the first and third equations of (9), the normal system

$$
\begin{aligned}
\bar{x}_{1}(k+1) & =\widetilde{A} \bar{x}_{1}(k)+\widetilde{B} u(k)+\widetilde{G}_{1} d(k), \\
y(k) & =\widetilde{C} \bar{x}_{1}(k)+\widetilde{D} u(k)+\widetilde{G}_{2} d(k)
\end{aligned}
$$

is obtained, where

$$
\begin{aligned}
\widetilde{A} & =\bar{A}_{11}-\bar{A}_{12} \bar{A}_{22}^{-1} \bar{A}_{21} \\
\widetilde{B} & =\bar{B}_{1}-\bar{A}_{12} \bar{A}_{22}^{-1} \bar{B}_{2}, \\
\widetilde{G}_{1} & =\bar{G}_{1}-\bar{A}_{12} \bar{A}_{22}^{-1} \bar{G}_{2}, \\
\widetilde{C} & =\bar{C}_{1}-\bar{C}_{2} \bar{A}_{22}^{-1} \bar{A}_{21}, \\
\widetilde{D} & =-\bar{C}_{2} \bar{A}_{22}^{-1} \bar{B}_{2}+D, \\
\widetilde{G}_{2} & =-\bar{C}_{2} \bar{A}_{22}^{-1} \bar{G}_{2} .
\end{aligned}
$$

\section{Main Theorems and the Proofs}

Since the limited equivalent transformation does not change the dynamic characteristics, we only need to design a preview controller for (14). The error system method is still needed. First, the error system is constructed. Then, the controller is designed according to the results of the optimal preview control theory [20].

Taking the first-order forward difference operator $\Delta$,

$$
\Delta v(k)=v(k+1)-v(k) .
$$

Define the new state vector $X(k)=\left[\begin{array}{c}e(k) \\ \Delta \bar{x}_{1}(k)\end{array}\right]$, and the error system is

$$
\begin{aligned}
X(k+1)= & \widehat{A} X(k)+\widehat{B} \Delta u(k)+\widehat{G}_{d 0} \Delta d(k) \\
& +\widehat{G}_{r 0} \Delta r(k), \\
e(k)= & \widehat{C} X(k),
\end{aligned}
$$

where

$$
\begin{aligned}
\widehat{A} & =\left[\begin{array}{cc}
I_{m} & \widetilde{C} \\
0 & \widetilde{A}
\end{array}\right], \\
\widehat{B} & =\left[\begin{array}{c}
\widetilde{D} \\
\widetilde{B}
\end{array}\right], \\
\widehat{G}_{d 0} & =\left[\begin{array}{c}
\widetilde{G}_{2} \\
\widetilde{G}_{1}
\end{array}\right], \\
\widehat{G}_{r 0} & =\left[\begin{array}{c}
-I_{m} \\
0
\end{array}\right], \\
\widehat{C} & =\left[\begin{array}{ll}
I_{m} & 0
\end{array}\right] .
\end{aligned}
$$


Remark 8. Since the reference signal $r(k)$ and the output $y(k)$ are both known, it is reasonable to take the error signal $e(k)=$ $y(k)-r(k)$ as the output of (17), spontaneously.

Expressing the performance index (6) with the relevant terms in (17), (6) can be written as

$$
J=\sum_{k=1}^{\infty}\left[X^{T}(k) Q X(k)+\Delta u^{T}(k) H \Delta u(k)\right],
$$

where $Q=\left[\begin{array}{cc}Q_{e} & 0 \\ 0 & 0\end{array}\right]=\widehat{C}^{T} Q_{e} \widehat{C}$. The main results of this paper can be gained immediately by the approach similar to [20].

Theorem 9. Assume that the following conditions are satisfied:

(1) $(\widetilde{A}, \widetilde{B})$ is stabilizable and the matrix $\left[\begin{array}{cc}I_{q}-\widetilde{A} & \widetilde{B} \\ -\widetilde{C} & \widetilde{D}\end{array}\right]$ is of full row rank.

(2) $(\widetilde{C}, \widetilde{A})$ is detectable.

(3) $Q_{e}>0$.

Then the optimal controller of (17) which can minimize the performance index function (19) is

$\Delta u(k)$

$$
\begin{aligned}
& =F_{0} X(k)+\sum_{j=0}^{M_{r}} F_{r}(j) \Delta r(k+j) \\
& \quad+\sum_{j=0}^{M_{d}} F_{d}(j) \Delta d(k+j) \\
& =F_{e} e(k)+F_{\bar{x}_{1}} \Delta \bar{x}_{1}(k) \\
& \quad+\sum_{j=0}^{M_{r}} F_{r}(j) \Delta r(k+j)+\sum_{j=0}^{M_{d}} F_{d}(j) \Delta d(k+j),
\end{aligned}
$$

where

$$
\begin{aligned}
& F_{0}=\left[\begin{array}{ll}
F_{e} & F_{\bar{x}_{1}}
\end{array}\right]=-\left[H+\widehat{B}^{T} P \widehat{B}\right]^{-1} \widehat{B}^{T} P \widehat{A}, \\
& F_{r}(j)=-\left[H+\widehat{B}^{T} P \widehat{B}\right]^{-1} \widehat{B}^{T}\left(\xi^{T}\right)^{j} P \widehat{G}_{r 0} \\
& \left(j=0,1,2, \ldots, M_{r}\right), \\
& F_{d}(j)=-\left[H+\widehat{B}^{T} P \widehat{B}\right]^{-1} \widehat{B}^{T}\left(\xi^{T}\right)^{j} P \widehat{G}_{d 0} \\
& \left(j=0,1,2, \ldots, M_{d}\right), \\
& \xi=\widehat{A}+\widehat{B} F_{0} .
\end{aligned}
$$

$P \geq 0$ is the unique semipositive solution of the following algebraic Riccati equation:

$$
P=Q+\widehat{A}^{T} P \widehat{A}-\widehat{A}^{T} P \widehat{B}\left[H+\widehat{B}^{T} P \widehat{B}^{-1} \widehat{B}^{T} P \widehat{A} .\right.
$$

According to Lemma 2 in [20], if $(\widetilde{A}, \widetilde{B})$ is stabilizable, $(\widetilde{C}, \widetilde{A})$ is detectable, and the matrix $\left[\begin{array}{cc}I_{q}-\widetilde{A} & \widetilde{B} \\ -\widetilde{C} & \widetilde{D}\end{array}\right]$ is of full row rank; there exists a unique semipositive solution for the Riccati equation (22). In the following, the conditions satisfying Theorem 9 are given.

Firstly, the conditions that ensure that $(\widetilde{A}, \widetilde{B})$ is stabilizable are studied.

Lemma 10. $(\widetilde{A}, \widetilde{B})$ is stabilizable if and only if $(E, A, B)$ is stabilizable.

Proof. Because the limited equivalence transformation keeps the stabilizability of the system $[11]$, so $(\bar{E}, \bar{A}, \bar{B})$ is stabilizable if and only if $(E, A, B)$ is stabilizable.

Then, according to the $\mathrm{PBH}$ criterion, $(\bar{E}, \bar{A}, \bar{B})$ is stabilizable if and only if, for any complex $z$ satisfying $|z| \geq 1$, the matrix

$$
\Psi=\left[z\left[\begin{array}{ll}
I_{q} & 0 \\
0 & 0
\end{array}\right]-\left[\begin{array}{ll}
\bar{A}_{11} & \bar{A}_{12} \\
\bar{A}_{21} & \bar{A}_{22}
\end{array}\right] \mid\left[\begin{array}{l}
\bar{B}_{1} \\
\bar{B}_{2}
\end{array}\right]\right]
$$

is of full row rank. Due to $\bar{A}_{22}$ being nonsingular,

$$
\begin{aligned}
& \Psi \longrightarrow \\
& {\left[\begin{array}{cc|c}
z I_{q}-\left(\bar{A}_{11}-\bar{A}_{12} \bar{A}_{22}-\bar{A}_{21}\right) & 0 & \bar{B}_{1}-\bar{A}_{12} \bar{A}_{22}^{-1} \bar{B}_{2} \\
0 & -\bar{A}_{22} & 0
\end{array}\right]} \\
& \quad=\left[\begin{array}{cc|c}
z I_{q}-\widetilde{A} & 0 & B \\
0 & -\bar{A}_{22} & 0
\end{array}\right]
\end{aligned}
$$

can be obtained by primary transformation. Therefore, $\Psi$ is of full row rank if and only if $\left[z I_{q}-\widetilde{A} \widetilde{B}\right]$ is of full row rank. That is to say, $(\widetilde{A}, \widetilde{B})$ is stabilizable if and only if $(\bar{E}, \bar{A}, \bar{B})$ is stabilizable. This accomplishes the proof.

Lemma 11. Matrix $\left[\begin{array}{ccc}I_{q}-\widetilde{A} & \widetilde{B} \\ -\widetilde{C} & \widetilde{D}\end{array}\right]$ is of full row rank if and only if $\left[\begin{array}{cc}E-A & B \\ -C & D\end{array}\right]$ is of full row rank.

Proof. Since

$$
\begin{aligned}
& {\left[\begin{array}{cc}
I_{q}-\widetilde{A} & \widetilde{B} \\
-\widetilde{C} & \widetilde{D}
\end{array}\right]} \\
& \quad=\left[\begin{array}{cc}
I_{q}-\left(\bar{A}_{11}-\bar{A}_{12} \bar{A}_{22}^{-1} \bar{A}_{21}\right) & B_{1}-\bar{A}_{12} \bar{A}_{22}^{-1} B_{2} \\
-\bar{C}_{1}+\bar{C}_{2} \bar{A}_{22}^{-1} A_{21} & D-\bar{C}_{2} \bar{A}_{22}^{-1} B_{2}
\end{array}\right]
\end{aligned}
$$

by using the nonsingular matrices $P_{1}$ and $Q_{1}$, we have

$$
\begin{gathered}
\operatorname{rank}\left[\begin{array}{cc}
Q_{1} & 0 \\
0 & I
\end{array}\right]\left[\begin{array}{cc}
E-A & B \\
-C & D
\end{array}\right]\left[\begin{array}{cc}
P_{1} & 0 \\
0 & I
\end{array}\right] \\
=\operatorname{rank}\left[\begin{array}{cc}
Q_{1} E P_{1}-Q_{1} A P_{1} & Q_{1} B \\
-C P_{1} & D
\end{array}\right] .
\end{gathered}
$$


Furthermore,

$$
\begin{aligned}
& {\left[\begin{array}{cc}
Q_{1} E P_{1}-Q_{1} A P_{1} & Q_{1} B \\
-C P_{1} & D
\end{array}\right]} \\
& \quad=\left[\begin{array}{cc}
{\left[\begin{array}{ll}
I_{q} & 0 \\
0 & 0
\end{array}\right]-\left[\begin{array}{cc}
\bar{A}_{11} & \bar{A}_{12} \\
\bar{A}_{21} & \bar{A}_{22}
\end{array}\right]} & \bar{B}_{1} \\
{\left[\begin{array}{c}
-\bar{C}_{1} \\
-\bar{C}_{2}
\end{array}\right]} & D
\end{array}\right] \\
& \quad=\left[\begin{array}{ccc}
I_{q}-\bar{A}_{11} & -\bar{A}_{12} & \bar{B}_{1} \\
-\bar{A}_{21} & -\bar{A}_{22} & \bar{B}_{2} \\
-\bar{C}_{1} & -\bar{C}_{2} & D
\end{array}\right] .
\end{aligned}
$$

$$
\begin{array}{r}
\operatorname{rank}\left[\begin{array}{cc}
Q_{1} & 0 \\
0 & I
\end{array}\right]\left[\begin{array}{cc}
E-A & B \\
-C & D
\end{array}\right]\left[\begin{array}{cc}
P_{1} & 0 \\
0 & I
\end{array}\right] \\
=\operatorname{rank}\left[\begin{array}{ccc}
I_{q}-\bar{A}_{11} & -\bar{A}_{12} & \bar{B}_{1} \\
-\bar{A}_{21} & -\bar{A}_{22} & \bar{B}_{2} \\
-\bar{C}_{1} & -\bar{C}_{2} & D
\end{array}\right] .
\end{array}
$$

Since $\left[\begin{array}{cc}Q_{1} & 0 \\ 0 & I\end{array}\right]$ and $\left[\begin{array}{cc}P_{1} & 0 \\ 0 & I\end{array}\right]$ are both nonsingular, so

$$
\operatorname{rank}\left[\begin{array}{cc}
E-A & B \\
-C & D
\end{array}\right]=\operatorname{rank}\left[\begin{array}{ccc}
I_{q}-\bar{A}_{11} & -\bar{A}_{12} & \bar{B}_{1} \\
-\bar{A}_{21} & -\bar{A}_{22} & \bar{B}_{2} \\
-\bar{C}_{1} & -\bar{C}_{2} & D
\end{array}\right] \text {. }
$$

Because

$$
\begin{gathered}
{\left[\begin{array}{lll}
I & 0 & 0 \\
0 & 0 & I \\
0 & I & 0
\end{array}\right]\left[\begin{array}{ccc}
I & -\bar{A}_{12} \bar{A}_{22}^{-1} & 0 \\
0 & I & 0 \\
0 & 0 & I
\end{array}\right]\left[\begin{array}{ccc}
I_{q}-\bar{A}_{11} & -\bar{A}_{12} & \bar{B}_{1} \\
-\bar{A}_{21} & -\bar{A}_{22} & \bar{B}_{2} \\
-\bar{C}_{1} & -\bar{C}_{2} & D
\end{array}\right]\left[\begin{array}{ccc}
I & 0 & 0 \\
\bar{A}_{22}^{-1} \bar{A}_{21} & I & 0 \\
0 & 0 & I
\end{array}\right]\left[\begin{array}{lll}
I & 0 & 0 \\
0 & 0 & I \\
0 & I & 0
\end{array}\right]} \\
\quad=\left[\begin{array}{ccc}
I_{q}-\left(\bar{A}_{11}-\bar{A}_{12} \bar{A}_{22}^{-1} \bar{A}_{21}\right) & B_{1}-\bar{A}_{12} \bar{A}_{22}^{-1} \bar{B}_{2} & 0 \\
-\bar{C}_{1}+\bar{C}_{2} \bar{A}_{22}^{-1} \bar{A}_{21} & D-\bar{C}_{2} \bar{A}_{22}^{-1} \bar{B}_{2} & 0 \\
0 & 0 & -\bar{A}_{22}
\end{array}\right]=\left[\begin{array}{ccc}
I_{q}-\widetilde{A} & \widetilde{B} & 0 \\
-\widetilde{C} & \widetilde{D} & 0 \\
\hline 0 & 0 & -\bar{A}_{22}
\end{array}\right]
\end{gathered}
$$

and the matrices $\left[\begin{array}{lll}I & 0 & 0 \\ 0 & 0 & I \\ 0 & I & 0\end{array}\right],\left[\begin{array}{ccc}I & -\bar{A}_{12} \bar{A}_{22}^{-1} & 0 \\ 0 & I & 0 \\ 0 & 0 & I\end{array}\right]$, and $\left[\begin{array}{ccc}I & 0 & 0 \\ \bar{A}_{22}^{-1} \bar{A}_{21} & I & 0 \\ 0 & 0 & I\end{array}\right]$ are all nonsingular, we have

$$
\begin{aligned}
& \operatorname{rank}\left[\begin{array}{ccc}
I_{q}-\bar{A}_{11} & -\bar{A}_{12} & \bar{B}_{1} \\
-\bar{A}_{21} & -\bar{A}_{22} & \bar{B}_{2} \\
-\bar{C}_{1} & -\bar{C}_{2} & D
\end{array}\right] \\
& =\operatorname{rank}\left[\begin{array}{cc|c}
I_{q}-\widetilde{A} & \widetilde{B} & 0 \\
-\widetilde{C} & \widetilde{D} & 0 \\
\hline 0 & 0 & -\bar{A}_{22}
\end{array}\right] .
\end{aligned}
$$

According to (29) and (31), we have

$$
\operatorname{rank}\left[\begin{array}{cc|c}
I_{q}-\widetilde{A} & \widetilde{B} & 0 \\
-\widetilde{C} & \widetilde{D} & 0 \\
\hline 0 & 0 & -\bar{A}_{22}
\end{array}\right]=\operatorname{rank}\left[\begin{array}{cc}
E-A & B \\
-C & D
\end{array}\right] \text {. }
$$

Because $\bar{A}_{22}$ is a nonsingular matrix, the matrix $\left[\begin{array}{ccc}I_{q}-\widetilde{A} & \widetilde{B} \\ -\widetilde{C} & \widetilde{D}\end{array}\right]$ is of full row rank if and only if $\left[\begin{array}{ccc}E-A & B \\ -C & D\end{array}\right]$ is of full row rank. This accomplishes the proof.

Secondly, the conditions that ensure that $(\widetilde{C}, \widetilde{A})$ is detectable are studied.
Lemma 12. $(\widetilde{C}, \widetilde{A})$ is detectable if and only if $(E, A, C)$ is detectable.

Proof. Because the limited equivalence transformation keeps the detectability of a system unchanged [11], $(\bar{E}, \bar{A}, \bar{C})$ is detectable if and only if $(E, A, C)$ is detectable.

According to the $\mathrm{PBH}$ criterion, $(\bar{E}, \bar{A}, \bar{C})$ is detectable if and only if, for any complex $z$ satisfying $|z| \geq 1$, the matrix

$$
\begin{aligned}
& \Omega=\left[\begin{array}{c}
z\left[\begin{array}{cc}
I_{q} & 0 \\
0 & 0
\end{array}\right]-\left[\begin{array}{ll}
\bar{A}_{11} & \bar{A}_{12} \\
\bar{A}_{21} & \bar{A}_{22}
\end{array}\right] \\
{\left[\bar{C}_{1} \bar{C}_{2}\right]}
\end{array}\right] \\
& =\left[\begin{array}{cc}
z I_{q}-\bar{A}_{11} & -\bar{A}_{12} \\
-\bar{A}_{21} & -\bar{A}_{22} \\
\vec{C}_{1} & \bar{C}_{2}
\end{array}\right]
\end{aligned}
$$

is of full column rank. Due to $\bar{A}_{22}$ being nonsingular,

$$
\begin{aligned}
& \Omega \longrightarrow \\
& {\left[\begin{array}{cc}
z I_{q}-\left(\bar{A}_{11}-\bar{A}_{12} \bar{A}_{22}^{-1} \bar{A}_{21}\right) & 0 \\
\bar{C}_{1}-\bar{C}_{2} \bar{A}_{22}^{-1} \bar{A}_{21} & 0 \\
0 & -\bar{A}_{22}
\end{array}\right]} \\
& =\left[\begin{array}{cc}
z I_{q}-\widetilde{A} & 0 \\
\widetilde{C} & 0 \\
0 & -\bar{A}_{22}
\end{array}\right]
\end{aligned}
$$


can be obtained by primary transformation. Then $\Omega$ is of full column rank if and only if $\left[\begin{array}{c}z I_{q}-\widetilde{A} \\ \widetilde{C}\end{array}\right]$ is of full column rank. That is to say, $(\widetilde{C}, \widetilde{A})$ is detectable if and only if $(\bar{E}, \bar{A}, \bar{C})$ is detectable. This accomplishes the proof.

Then, we can get the following theorem.

Theorem 13. If Assumptions 1, 2, 3, and 4 and $Q_{e}>0$ hold, the optimal preview controller for system (1) is

$$
\begin{aligned}
u(k)= & u(0)+F_{e} \sum_{i=0}^{k-1} e(i)+F_{x} x(k)-F_{x} x(0) \\
& +\sum_{j=0}^{M_{r}} \sum_{i=1}^{k} F_{r}(j) \Delta r(j+i-1) \\
& +\sum_{j=0}^{M_{d}} \sum_{i=1}^{k} F_{d}(j) \Delta d(j+i-1),
\end{aligned}
$$

where $F_{x}=\left[\begin{array}{ll}F_{\bar{x}_{1}} & 0\end{array}\right] P_{1}^{-1}$, and the other coefficient matrices are determined by (21)-(22). $\bar{x}_{1}(0)$ and $u(0)$ can be assigned to any value. $x(0)=P_{1}\left[\begin{array}{l}\bar{x}_{1}(0) \\ \bar{x}_{2}(0)\end{array}\right] \cdot \bar{x}_{2}(k)$ can be determined by (13).

Proof. If Assumptions 1, 2, 3, and 4 and $Q_{e}>0$ hold, the conditions of Theorem 9 are all satisfied. Then, the optimal controller for system (17) is (20). In order to prove this theorem, we only need to get (35) by (20).

Since $\Delta u(k-1)=u(k)-u(k-1)$ and $\Delta \bar{x}_{1}(k-1)=\bar{x}_{1}(k)-$ $\bar{x}_{1}(k-1)$, based on $(20)$, we can get

$$
\begin{aligned}
& u(1)-u(0)=F_{e} e(0)+F_{\bar{x}_{1}}\left[\bar{x}_{1}(1)-\bar{x}_{1}(0)\right] \\
& +\sum_{j=0}^{M_{r}} F_{r}(j) \Delta r(j)+\sum_{j=0}^{M_{d}} F_{d}(j) \Delta d(j), \\
& u(2)-u(1)=F_{e} e(1)+F_{\bar{x}_{1}}\left[\bar{x}_{1}(2)-\bar{x}_{1}(1)\right] \\
& +\sum_{j=0}^{M_{r}} F_{r}(j) \Delta r(j+1)+\sum_{j=0}^{M_{d}} F_{d}(j) \Delta d(j+1), \\
& \quad \vdots \\
& +F_{\bar{x}_{1}}\left[\bar{x}_{1}(k)-\bar{x}_{1}(k-1)\right] \\
& +\sum_{j=0}^{M_{r}} F_{r}(j) \Delta r(j+k-1) \\
& +\sum_{j=0}^{M_{d}} F_{d}(j) \Delta d(j+k-1) .
\end{aligned}
$$

The sum of the above equations can be written as

$$
\begin{aligned}
u(k)-u(0)= & F_{e} \sum_{i=0}^{k-1} e(i)+F_{\bar{x}_{1}} \bar{x}_{1}(k)-F_{\bar{x}_{1}} \bar{x}_{1}(0) \\
& +\sum_{j=0}^{M_{r}} \sum_{i=1}^{k} F_{r}(j) \Delta r(j+i-1) \\
& +\sum_{j=0}^{M_{d}} \sum_{i=1}^{k} F_{d}(j) \Delta d(j+i-1) ;
\end{aligned}
$$

that is,

$$
\begin{aligned}
u(k)= & u(0)+F_{e} \sum_{i=0}^{k-1} e(i)+F_{\bar{x}_{1}} \bar{x}_{1}(k)-F_{\bar{x}_{1}} \bar{x}_{1}(0) \\
& +\sum_{j=0}^{M_{r}} \sum_{i=1}^{k} F_{r}(j) \Delta r(j+i-1) \\
& +\sum_{j=0}^{M_{d}} \sum_{i=1}^{k} F_{d}(j) \Delta d(j+i-1) .
\end{aligned}
$$

Because $x(k)=P_{1} \bar{x}(k)$ and $\bar{x}(k)=\left[\begin{array}{l}\bar{x}_{1}(k) \\ \bar{x}_{2}(k)\end{array}\right],\left[\begin{array}{l}\bar{x}_{1}(k) \\ \bar{x}_{2}(k)\end{array}\right]=$ $P_{1}^{-1} x(k)$ can be obtained. Then, $F_{\bar{x}_{1}} \bar{x}_{1}(k)=\left[\begin{array}{ll}F_{\bar{x}_{1}} & 0\end{array}\right]\left[\begin{array}{l}\bar{x}_{1}(k) \\ \bar{x}_{2}(k)\end{array}\right]=$ $\left[F_{\bar{x}_{1}} 0\right] P_{1}^{-1} x(k)=F_{x} x(k)$. When $k=0, F_{\bar{x}_{1}} \bar{x}_{1}(0)=F_{x} x(0)$. Substituting $F_{\bar{x}_{1}} \bar{x}_{1}(k)$ and $F_{\bar{x}_{1}} \bar{x}_{1}(0)$ into (38), we have (35).

This completes the proof.

By observing (35), we can find that the term $F_{e} \sum_{i=0}^{k} e(i)$ is included in $u(k)$, which leads to an integrator contained in the corresponding closed-loop system. This is originated from the introduction of $\Delta u(k)$ in the performance index (6). In addition, $\sum_{j=0}^{M_{r}} \sum_{i=1}^{k} F_{r}(j) \Delta r(j+i-1)$ and $\sum_{j=0}^{M_{d}} \sum_{i=1}^{k} F_{d}(j) \Delta d(j+i-1)$ in (35) are the previewable reference signal and disturbance signal, respectively.

\section{Study on the Numerical Simulation Method}

In the following, we need to deal with the numerical simulation problem with the state equation in system (1); that is,

$$
E x(k+1)=A x(k)+B u(k)+G d(k),
$$

and with the controller $u(k)$ in (35). Since the singular matrix $E$ is included in (39), the state vector $x(k+1)$ cannot be calculated directly when simulating.

A new method needs to be designed to solve this problem.

Firstly, an appropriate matrix $M$ is selected to make $E+M$ be nonsingular.

Secondly, adding the identical equation

$$
M x(k+1)=M x(k+1)
$$

to (39), we have

$$
\begin{aligned}
(E+M) x(k+1)= & M x(k+1)+A x(k)+B u(k) \\
& +G d(k) .
\end{aligned}
$$


Namely,

$$
\begin{aligned}
x(k+1)= & (E+M)^{-1} M x(k+1) \\
& +(E+M)^{-1}[A x(k)+B u(k)+G d(k)] .
\end{aligned}
$$

Equation (42) is still unable to be calculated because the term $x(k+1)$ is on the right side of the equation. To tackle this problem, we take $x(k)$ as the approximate value of $x(k+1)$ on the right side of (42); that is,

$$
\begin{aligned}
x(k+1)= & (E+M)^{-1} M x(k) \\
& +(E+M)^{-1}[A x(k)+B u(k)+G d(k)] .
\end{aligned}
$$

In this way, the simulation can be carried out.

It is obvious that the above iterative method is equal to adding the term $M x(k+1)$ to the left side and the term $M x(k)$ to the right side of (39), essentially. This iterative method is reasonable: if the output of the closed-loop system is able to track the reference signal, there exist $x(\infty), u(\infty)$, and $d(\infty)$ such that

$$
\begin{aligned}
E x(\infty) & =A x(\infty)+B u(\infty)+G d(\infty) \\
y(\infty) & =C x(\infty)+D u(\infty) .
\end{aligned}
$$

Meanwhile, if the iterative method (43) is convergent, the same relation can be obtained by letting $k \rightarrow \infty$ on both sides of (43) and on the observation equation of system (1). In other words, when $k$ is very large, $M x(k+1) \approx M x(k)$. Then, the solution of (43) is quite close to the solution of (39).

Thirdly, the convergent condition for the iterative method (43) is gained. Note that

$$
u(k)=F_{x} x(k)+f(k)
$$

where

$$
\begin{aligned}
f(k)= & u(0)+F_{e} \sum_{i=0}^{k} e(i)-F_{x} x(0) \\
& +\sum_{j=0}^{M_{r}} \sum_{i=1}^{k} F_{r}(j) \Delta r(j+i-1) \\
& +\sum_{j=0}^{M_{d}} \sum_{i=1}^{k} F_{d}(j) \Delta d(j+i-1) .
\end{aligned}
$$

Substituting $u(k)$ into (43), we have the closed-loop system

$$
\begin{aligned}
x(k+1)= & (E+M)^{-1}\left(M+A+B F_{x}\right) x(k) \\
& +(E+M)^{-1}[B f(k)+G d(k)] .
\end{aligned}
$$

Since $(E+M)^{-1}[B f(k)+G d(k)]$ is a small perturbation part, a sufficient condition which ensures that the iterative method (43) is convergent is that the spectral radius of $(E+$ $M)^{-1}\left(M+A+B F_{x}\right)$ (i.e., the maximum value of the absolute value of the eigenvalues) is less than 1 [21].
In conclusion, if there exists an appropriate matrix $M$ which makes $E+M$ be nonsingular and the spectral radius of $(E+M)^{-1}\left(M+A+B F_{x}\right)$ be less than 1 , the output response of the closed-loop system of (1) can be obtained by the iterative method (43) and by the output equation in (1), where the controller $u(k)$ is determined by (35).

The conclusions of this section can be applied to the numerical simulation of all discrete-time descriptor systems.

\section{Simulation Example}

Consider system (1) with coefficient matrices

$$
\begin{aligned}
E & =\left[\begin{array}{cccc}
0.75 & 0.75 & 0 & 0.75 \\
1.5 & 0.75 & 0.75 & 0 \\
1.5 & 1.5 & 0 & 1.5 \\
1.5 & 0.75 & 1.5 & 0.75
\end{array}\right], \\
A & =\left[\begin{array}{cccc}
4 & 1 & 1 & 0 \\
-1 & 1 & -2 & 0 \\
1 & -1 & 2 & 1 \\
-1 & 2 & 20 & 1
\end{array}\right],
\end{aligned}
$$

$$
B=\left[\begin{array}{l}
0 \\
0 \\
0 \\
1
\end{array}\right] \text {, }
$$

$$
G=\left[\begin{array}{l}
0 \\
1 \\
1 \\
0
\end{array}\right],
$$

$$
\begin{aligned}
& C=\left[\begin{array}{llll}
1 & 0 & 0 & 0
\end{array}\right], \\
& D=0 .
\end{aligned}
$$

Let the initial state vector be $x(0)=\left[\begin{array}{llll}0 & 0 & 0 & 0\end{array}\right]^{T}$, the initial input vector be $u(0)=0$, the reference signal be

$$
r(k)= \begin{cases}0, & 0<k<50 \\ 1, & k \geq 50\end{cases}
$$

and the interference signal be

$$
d(k)= \begin{cases}0, & 0<k<300, \\ \frac{1}{2}, & k \geq 300 .\end{cases}
$$

Take the weight matrices

$$
\begin{gathered}
Q_{e}=1, \\
H=1 .
\end{gathered}
$$




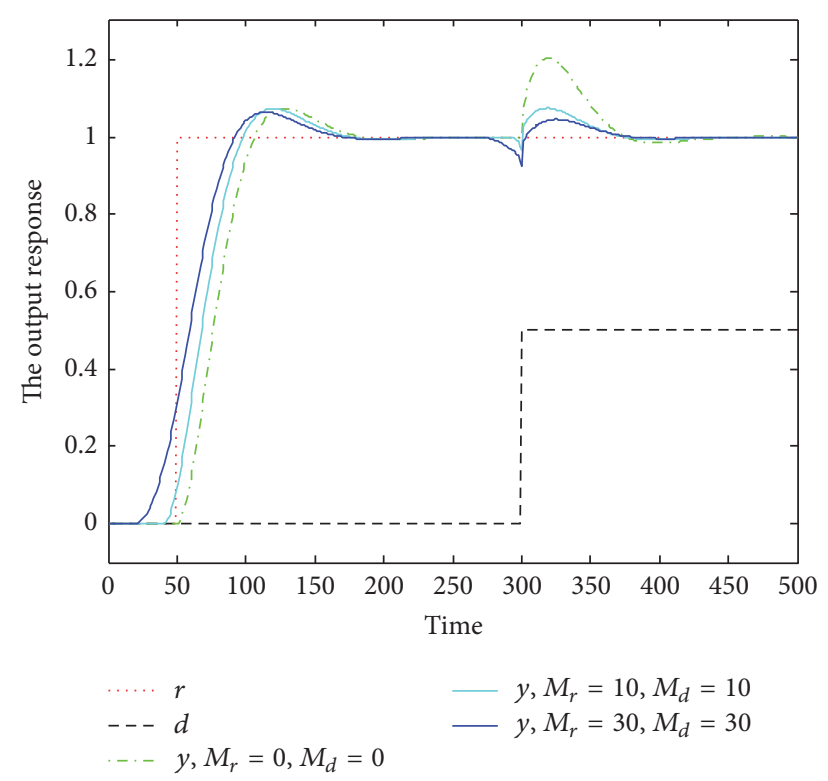

FIGURE 1: The output response to step function with preview.

By calculating we can know that Assumptions 1, 2, 3, and 4 can be satisfied with the above system. That is, all the conditions of Theorem 13 are satisfied.

Let $M=\left[\begin{array}{cccc}-3.89 & -1 & -1 & 0 \\ 1 & -0.89 & 2 & 0 \\ -1 & 1 & -1.89 & -1 \\ 43.58 & 17.23 & 2.01 & -6.33\end{array}\right]$. Then $E+M$ is nonsingular and the spectral radius of $(E+M)^{-1}\left(M+A+B F_{x}\right)$ is less than 1 . Thus, the iterative method (43) is convergent. The output response is shown in Figure 1.

It can be seen from Figure 1 that when the preview control approach is used, the disturbance can be effectively suppressed, and the tracking effect is improved significantly. In fact, the disturbance can be further suppressed by adjusting the spectral radius of $(E+M)^{-1}\left(M+A+B F_{x}\right)$.

\section{Conclusion}

In this paper, the optimal preview controller for linear discrete-time descriptor systems is designed. Firstly, the descriptor system is transformed into a normal system by introducing the limited equivalent transformation. Then, by using a difference operator, the error system is constructed. The optimal preview controller is obtained according to the known conclusions of preview control theory. At the same time, the existence of the optimal preview controller with the basic assumptions is also proved strictly. More importantly, we solved the simulation problem and the simulation method is very effective.

\section{Conflicts of Interest}

The authors declare that they have no conflicts of interest.

\section{Acknowledgments}

This work was supported by the National Natural Science Foundation of China (Grant no. 61174209).

\section{References}

[1] D. S. Naidu, Optimal Control Systems, CRC Press, Boca Raton, Fla, USA, 2002.

[2] T. Tsuchiya and T. Egami, "Digital preview and predictive control," Sangyou Tosho, 1992.

[3] T. Katayama, T. Ohki, T. Inoue, and T. Kato, "Design of an optimal controller for a discrete-time system subject to previewable demand," International Journal of Control, vol. 41, no. 3, pp. 677-699, 1985.

[4] T. Katayama and T. Hirono, "Design of an optimal servomechanism with preview action and its dual problem," International Journal of Control, vol. 45, no. 2, pp. 407-420, 1987.

[5] L. Mianzo and H. Peng, "A unified Hamiltonian approach for LQ and $H_{\infty}$; preview control algorithms," Journal of Dynamic Systems, Measurement and Control, vol. 3, no. 3, pp. 365-369, 1999.

[6] A. Hazell and D. J. Limebeer, "An efficient algorithm for discrete-time $H_{\infty}$ preview control," Automatica, vol. 44, no. 9, pp. 2441-2448, 2008.

[7] A. Kojima, " $H_{\infty}$ controller design for preview and delayed systems," IEEETransactions on Automatic Control, vol. 60, no. 2, pp. 404-419, 2015.

[8] N. Wang, K. E. Johnson, and A. D. Wright, "FX-RLS-based feedforward control for LIDAR-enabled wind turbine load mitigation," IEEE Transactions on Control Systems Technology, vol. 20, no. 5, pp. 1212-1222, 2012.

[9] H. Tokutake, S. Okada, and S. Sunada, "Disturbance preview controller and its application to a small UAV," Transactions of the Japan Society for Aeronautical and Space Sciences, vol. 55, no. 1, pp. 76-78, 2012.

[10] C. Gohrle, A. Schindler, A. Wagner, and O. Sawodny, "Road profile estimation and preview control for low-bandwidth active suspension systems," IEEE/ASME Transactions on Mechatronics, vol. 20, no. 5, pp. 2299-2310, 2014.

[11] G. R. Duan, Analysis and Design of Descriptor Linear Systems, vol. 23 of Advances in Mechanics and Mathematics, Springer, New York, NY, USA, 2010.

[12] J. Yang, S. Zhong, and L. Xiong, "A descriptor system approach to non-fragile $H_{\infty}$ control for uncertain fuzzy neutral systems," Fuzzy Sets and Systems. An International Journal in Information Science and Engineering, vol. 160, no. 4, pp. 423-438, 2009.

[13] M. Alma and M. Darouach, "Adaptive observers design for a class of linear descriptor systems," Automatica. A Journal of IFAC, the International Federation of Automatic Control, vol. 50, no. 2, pp. 578-583, 2014.

[14] Z. Wang, Y. Shen, and X. Zhang, "Actuator fault estimation for a class of nonlinear descriptor systems," International Journal of Systems Science. Principles and Applications of Systems and Integration, vol. 45, no. 3, pp. 487-496, 2014.

[15] M. K. Gupta, N. K. Tomar, and S. Bhaumik, "Full- and reducedorder observer design for rectangular descriptor systems with unknown inputs," Journal of the Franklin Institute. Engineering and Applied Mathematics, vol. 352, no. 3, pp. 1250-1264, 2015.

[16] F. J. Bejarano, "Functional unknown input reconstruction of descriptor systems: application to fault detection," Automatica. A Journal of IFAC, the International Federation of Automatic Control, vol. 57, pp. 145-151, 2015.

[17] F. Liao, Z. Zhang, and Y. Zhang, "Reduced order of the Riccati equation and optimal preview control of singular systems," Journal of University of Science and Technology Beijing, vol. 31, no. 4, pp. 520-524, 2009. 
[18] M. Cao and F. Liao, "Design of an optimal preview controller for linear discrete-time descriptor systems with state delay," International Journal of Systems Science. Principles and Applications of Systems and Integration, vol. 46, no. 5, pp. 932-943, 2015.

[19] F. Liao, Z. Ren, M. Tomizuka, and J. Wu, "Preview control for impulse-free continuous-time descriptor systems," International Journal of Control, vol. 88, no. 6, pp. 1142-1149, 2015.

[20] F.-C. Liao and P. Chen, "Optimal preview control based on state observers for linear discrete-time systems," Beijing Keji Daxue Xuebao/Journal of University of Science and Technology Beijing, vol. 36, no. 3, pp. 390-398, 2014.

[21] D. Faires and R. L. Burden, Numerical Methods, Brooks Cole Publishing Co., Pacific Grove, CA, Washington, USA, Second edition, 2002. 


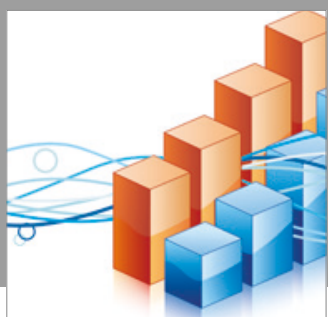

Advances in

Operations Research

vatersals

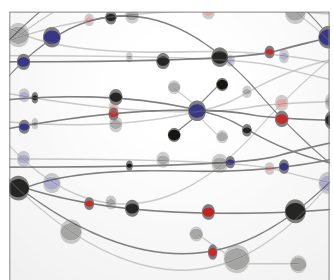

\section{The Scientific} World Journal
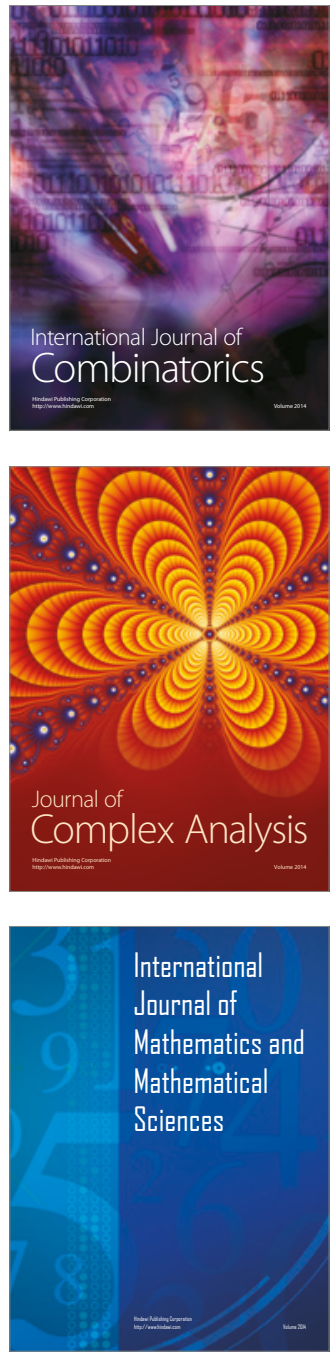
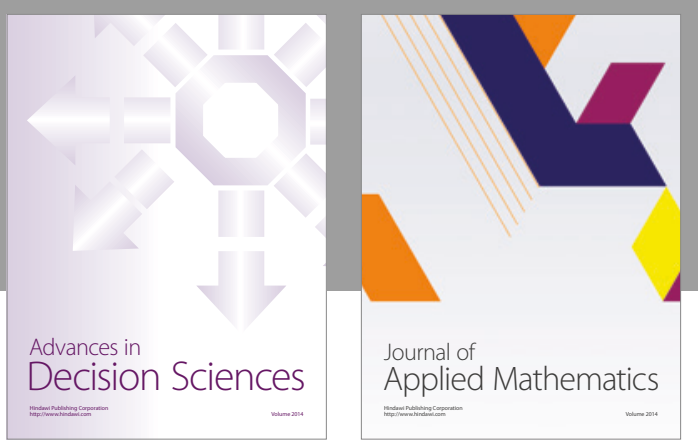

Algebra

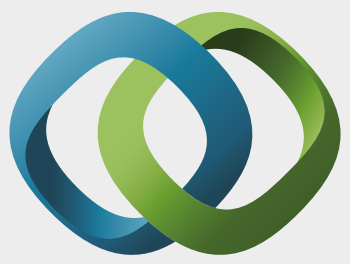

\section{Hindawi}

Submit your manuscripts at

https://www.hindawi.com
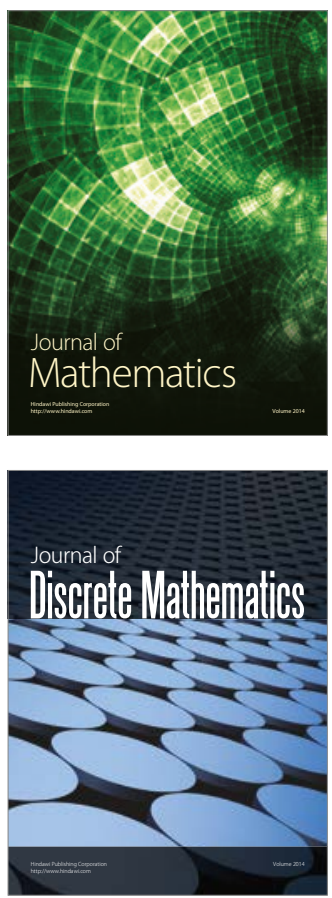

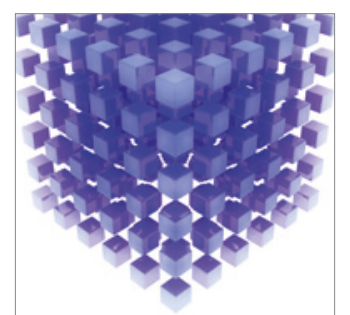

Mathematical Problems in Engineering
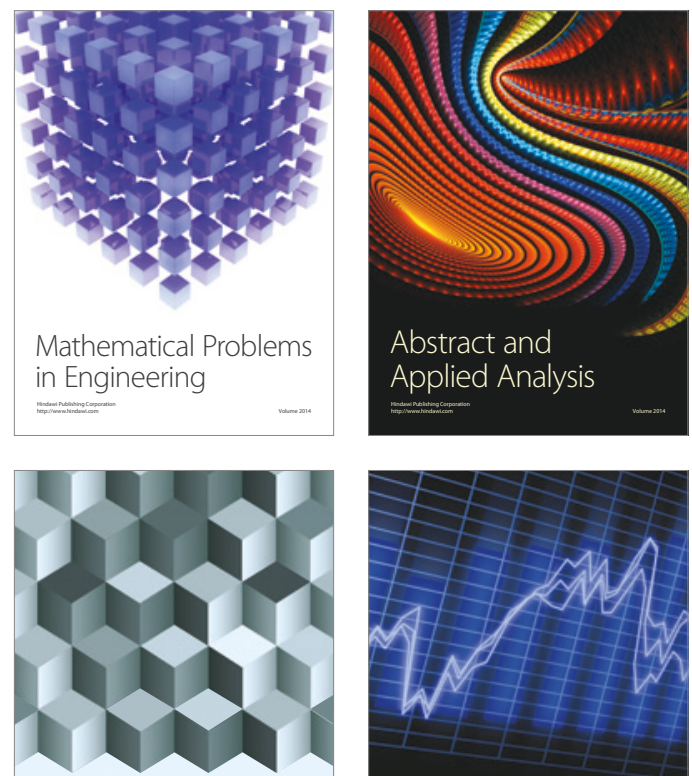

Journal of

Function Spaces

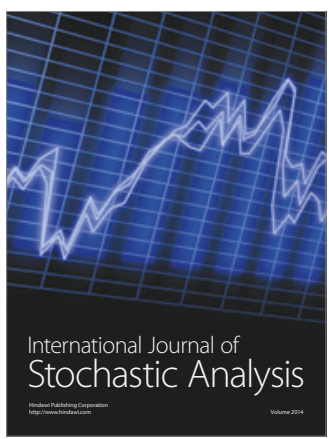

Probability and Statistics
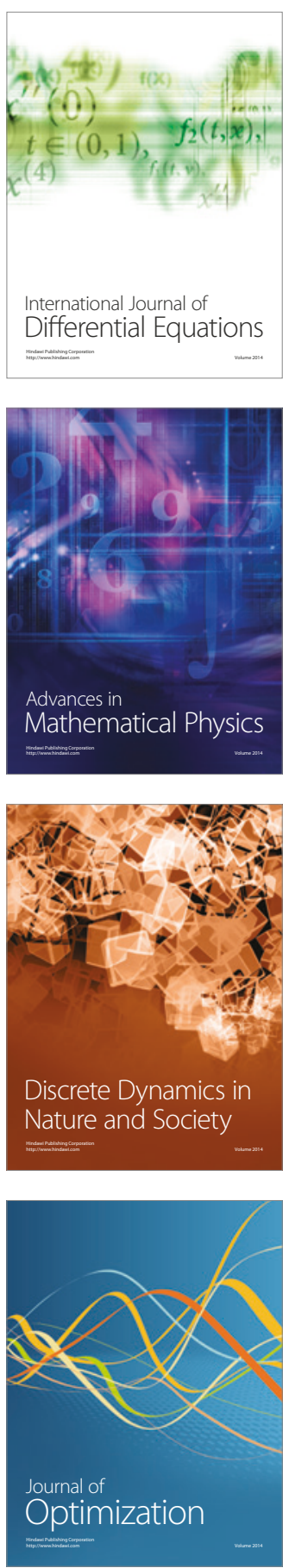